\title{
Bells and whistles of convection parameterization
}

Article

Accepted Version

Yano, J.-I., Machulskaya, E., Bechtold, P. and Plant, R. S. (2013) Bells and whistles of convection parameterization. Bulletin of the American Meteorological Society, 94 (1). ES5ES7. ISSN 1520-0477 doi: https://doi.org/10.1175/BAMS-D12-00118.1 Available at https://centaur.reading.ac.uk/28419/

It is advisable to refer to the publisher's version if you intend to cite from the work. See Guidance on citing.

To link to this article DOI: http://dx.doi.org/10.1175/BAMS-D-12-00118.1

Publisher: American Meteorological Society

All outputs in CentAUR are protected by Intellectual Property Rights law, including copyright law. Copyright and IPR is retained by the creators or other copyright holders. Terms and conditions for use of this material are defined in the End User Agreement.

\section{www.reading.ac.uk/centaur}

\section{CentAUR}

Central Archive at the University of Reading

Reading's research outputs online 
version:doc/workshop/savona/report: 6 June 2012

\title{
Bells and Whistles of Convection Parameterization
}

byJ.-I. Yano, E. Machulskaya, P. Bechtold, and R. S. Plant

\begin{abstract}
Affiliations:
Yano - GAME-CNRM, CNRS, Météo-France, Toulouse, France; Machulskaya - DWD, Offenbach, Germany; Bechtold - ECMWF, Reading, UK; Plant - University of Reading, $\mathrm{UK}$;
\end{abstract}

\section{Correspondence Author:}

Jun-Ichi Yano, GAME-CNRM, CNRS, Météo-France, 42 Av. Coriolis, Toulouse, France E-mail: jun-ichi.yano@zmaw.de 


\section{Meeting Title:}

Workshop on Concepts for Convective Parameterizations in Large-Scale Models V: "Bulk or Spectrum?"

What:

Thirty-Eight scientists from 16 European countries and Israel met to discuss the fundamental theoretical bells and whistles of convection parameterization in atmospheric modelling. When: 20-22 March 2012

Where: Savona, Italy 
The present workshop constitutes the 5th in the annual series on "Concepts for Convective Parameterizations in Large-Scale Models". The purpose of the workshop series has been to discuss the fundamental theoretical issues of convection parameterization with a small number of European scientists. The workshop series has been funded by European Cooperation in the Field of Scientific and Technical Research (COST) Action ES0905.

The theme of the workshop for the year 2012 was decided from a main conclusion of the previous workshop, which focused on the convective organization problem, seeking a means for implementing such effects into convection parameterizations (Yano et al. 2012). As it turned out, in order to discuss this implementation issue in any concrete manner, we have first to know very well the bells and whistles of convection parameterizations. This was the purpose of the 5th workshop.

The title of the workshop is rather metaphorically tagged as "Bulk or Spectrum?", because this is a typical decision we have to face at the outset of any parameterization development. The following report discusses selected issues of bells and whistles addressed during the meeting.

\section{OPERATIONAL CONSTRAINTS}

From an operational point of view, there are some fundamental constraints on parameterizations: conservation of mass, energy, and momentum. Within these constraints one could in principle develop a parametrization in any manner as long as overall forecast skill is improved. In practice, however this is very difficult to achieve, and only schemes based on solid meteorological principles tend to perform in a satisfactory manner.

Furthermore, both climatology and perturbation prediction must be correct in forecast models. If a perturbation prediction of equatorial convectively-coupled waves were the only issue, for example, then a model without any convective parameterization may perform better, as has often been reported in the literature, but only at the expense of substantially deteriorating the climatology.

Inclusion of mid-troposphere humidity sensitivities into entrainment and detrainment 
formulations has recently contributed substantially to the model improvements. For exm-

ple, it has significantly contributed to the improvement in prediction of Madden-Julian oscillations (MJO). In the ECMWF model, the forecast skill of the MJO has improved by roughly 5 days over the last 10 years, which may be compared to an average improvement of 1-2 days per decade for midlatitude $500 \mathrm{hPa}$ height.

\section{EQUILIBRIUM OR TRIGGERING?}

A basic question in parameterization is: to what extent is an ensemble of convective systems in a statistical equilibrium with the large-scale atmospheric state versus being controlled by the local processes that trigger individual storms? A plausible measure of this distinction can be made by considering the timescale over which convection is removing conditional instability. If this is shorter than a few hours, the convection can respond rapidly to changes in the large-scale environment and may be in equilibrium. An estimate of the convective timescale, based on the ratio of CAPE to precipitation rate, shows that timescales shorter and longer than a few hours occur with comparable frequencies (Zimmer et al. 2011). The distribution of timescales was found to follow a power law, with no sharp distinction between the two regimes. This result suggests that these two distinguishable regimes are not simple dichotomies.

\section{BULK OR SPECTRUM?}

The starting point for understanding mass-flux parameterizations is the classical spectral formulation of Arakawa and Schubert (1974). This approach represents a subgrid-scale convective system as a spectrum of various types of convective elements. The convective elements are traditionally represented by a plume model. The bulk approximation, in turn, consists of reducing the spectrum to a single "bulk" convective plume. It is emphasized, however, that a bulk model does not follow immediately from averaging over the spectrum of plumes, although we may wish to conceptually interpret it in this manner. For example, the detrainment of condensate is problematic in the theory of a bulk model, and discussions revealed that this also presents practical problems for some models. 
An inspection of bulk model output suggests that a bulk model, even under uniform forcing, in practice generates a time series of different convective events. Thus, as a time series, a spectral-like behavior is reproduced with a bulk approach. As a clear limitation, the bulk approach cannot be used with sophisticated microphysics, and so an ultimate superiority of the spectrum approach is indisputable. Hence, although spectral methods were agreed to be an important research topic, no urgent need for moving towards a spectral approach in operational practice was identified.

\section{PDF}

In addition to the mass flux formulation, two alternative approaches for parameterization development are also examined. The first is the PDF (probability density function) approach which has been applied especially for describing non-convective clouds at the subgrid scale.

Unfortunately, a PDF-based scheme requires a huge amount of input if it is to be constructed properly. A certain truncation should be thus applied to simplify the scheme. For example, the approach called "assumed PDF" takes a parametric family of PDFs that is described by a small number of free parameters. Among the possible choices, a double Gaussian distribution appears to be most promising, and is described by five independent parameters. Furthermore, this number can be reduced to three based on large-eddy simulations and observational analyses.

Once a PDF family is chosen, the next question is to determine the input parameters, i.e., the moments of the PDF. A widely used strategy is to determine the second- and higher-order moments based on a turbulence theory, but accounting for turbulent mixing only. Much investigation is still required in order to include the "nonmixing" processes properly. For this purpose, it would be important to start with a basic principle: a generalization of the Liouville principle to a continuous medium (cf., Larson 2004).

\section{SIMILARITY THEORY}

Another alternative possibility is the similarity theory (or scaling law). This theory 
may be considered an established approach for boundary-layer turbulence. Thus, it is tempting to generalize it to deep moist convection. For this generalization, it is important to distinguish between local and non-local transport processes. Local transport processes are downgradient and so can be represented by a conventional eddy-diffusion approach. Although non-local transport processes are more complex, the main hypothesis with similarity theory is that they can nevertheless be represented by a universal function after the appropriate nondimensionalization.

The keys to success for a similarity approach to deep moist convection is to identify the appropriate scaling parameters for nondimensionalizations, and then to define a universal non-local transport vertical profile. Note that by formulating the convection parameterization problem in this manner, the mass-flux parameterization becomes a special case that retains only a particular form of the non-local terms. If a local transport term is then included in the similarity formulation, it simply turns into the popular idea of combining eddy diffusion with mass-flux. The similarity theory provides a framework that encompasses all these approaches.

Although the formal applicability of the similarity theory is limited only to equilibrium solutions, the concept can be generalized into time-dependent problems by taking a formal asymptotic expansion approach with an appropriate nondimensionalization of a system. However, this more general and robust principle, unfortunately becomes difficult to develop into an actual procedure once the number of variables and physical processes increases.

\section{STOCHASTICITY}

The inclusion of stochasticity into convection parameterization is an attractive option considering its inherent unpredictability. Stochasticity in convection parameterization may, for example, improve the performance of an ensemble prediction system (EPS) by adding perturbations to the ensemble. A current major problem with various EPS is that they do not produce enough spread in the Tropics. At ECMWF, in order to alleviate lack of spread to some extent, the total physics tendency is perturbed within one standard deviation of 
the predicted tendency every time step.

Moreover, as the model resolution increases, the size of the sub-grid convection ensemble decreases, and thus intuitively convection would become less predictable even under a grid-box scale ensemble average. However, in considering stochasticity in the highresolution limit, we must face a subtle conceptual issue. In order to define a probability distribution to describe the stochasticity, a "convective ensemble" must be well defined in a similar sense as the "environment" is well defined in the traditional parameterization framework under scale separation. However, the concept of the "environment" cannot be equated with the grid-box state in the high-resolution limit because a scale separation principle is no longer applicable. In the absence of a clearly-defined "environment" it becomes less clear how to define a "convective ensemble" without such a well-defined references sate.

\section{SCALING AND DOWNSCALING}

Finally, a possibility for exploiting scaling behavior (power laws) for the characterization of convection is considered. The basic premise here is to describe an output variable by a certain power of an input variable as well as the scales. Scaling behavior is seen observationally in various atmospheric phenomena, including convection. It can be an important factor, for example, in adjusting a parameterization against the model resolution. Such scaling behavior further provides a basis for downscalings that provide a fine-scale distribution of precipitation, for example.

Downscaling is a serious operational issue, for example, over the geographical area stretching from Southern France to Northern Italy (the region including Savona). The area is prone to flash flood events due to the steep orography facing the Mediterranean Sea and the small river catchments. The last presentation of the workshop devoted to the scaling and downscaling turned out to be an important reminder of why we need to improve convection parameterization in operational contexts. 


\section{References:}

Arakawa, A., and W. H. Schubert 1974: Interaction of a cumulus cloud ensemble with the large-scale environment, Part I. J. Atmos. Sci., 31, 674-701.

Larson, V. E., 2004: Prognostic equations for cloud fraction and liquid water, and their relation to filtered density functions. J. Atmos. Sci., 61, 338-351.

Yano, J.-I., H.-F. Graf, and F. Spineanu, 2012: Meeting report: Theoretical and operational implications of atmospheric convective organization. Bull. Amer. Meteor. Soc., doi: http://dx.doi.org/10.1175/BAMS-D-11-00178.1

Zimmer, M., Craig, G. C., Wernli, H. and Keil, C., 2011: Classification of precipitation events with a convective response timescale. Geophys. Res. Lett., 38, L05802, doi:10.1029/2010GL046199. 